\title{
gynécologie suisse SSGO - un sigle de qualité et de sécurité
}

\author{
La Société suisse de gynécologie et d'obstétrique est la principale organisation du pays en matière de \\ santé des femmes. La sécurité et la qualité sont le fondement de la pratique médicale de nos membres et \\ ces notions servent également de base à la présentation de notre société.
}

\section{Dr Eduard Vlajkovic, responsable du département médias et communication}

Informations, documents à télécharger et contacts: www.sggg.ch.

\begin{abstract}
Historique
1905: création de la Société de gynécologie et d'obstétrique de la Suisse romande. 1911: création de la Société de gynécologie et d'obstétrique de la Suisse alémanique. 1915: regroupement des deux sociétés qui restent néanmoins indépendantes et ne fusionneront qu'en 1964 pour former la Société suisse de gynécologie. Cette organisation s'est développée en ayant une double fonction de société scientifique pour la gynécologie et l'obstétrique, d'une part et, d'association professionnelle pour ses membres, d'autre part. Cette double casquette sert à promouvoir notre principale tâche, à savoir la sécurité et la qualité.
\end{abstract}

\section{gynécologie suisse aujourd'hui}

La nouvelle désignation gynécologie suisse SGGG / SSGO fait de notre société le principal interlocuteur pour la santé des femmes et elle est garante de compétence et de qualité, d'ouverture et d'intégration, de force et d'unité. Forte de quelque 1400 membres, elle sera présidée pour la première fois cette année par une femme.

Actuellement, les éléments suivants nous servent de base de gestion en matière de qualité:

\section{Guidelines, manuels et avis d'experts}

Notre société met à disposition des directives pour la pratique clinique (guidelines) et des recommandations (avis d'experts et manuels) sous forme électronique. Basées sur la médecine fondée sur des preuves, ces directives doivent permettre un accroissement de l'efficacité dans le domaine diagnostique et thérapeutique. La liberté du médecin reste néanmoins garantie. Lors de prises de décision qui concernent les patients, il importe d'appliquer les connaissances médicales de manière claire, raisonnable et consciencieuse. Les avis d'experts contiennent des recommandations sur des sujets d'actualité et peuvent être publiées très rapidement comme directives.

\section{Informations aux patientes}

Le travail fourni par gynécologie suisse en matière d'information préopératoire des patientes est unique. En collaboration avec l'Organisation suisse des patients et la FMH, 28 protocoles d'information standard ont été élaborés dans les trois langues nationales, afin de pouvoir donner aux patientes des informations juridiquement correctes et appropriées sur les interventions à venir.

\section{Formation continue contrôlée}

En s'affiliant à l'Académie de formation continue de la $S S G O$, chaque membre s'engage à accomplir un nombre minimum d'heures de formation continue par an. L'Académie se charge du contrôle de la formation et de l'attribution des crédits. Un ou une gynécologue accrédité-e par l'Académie doit effectuer au moins 80 heures de formation continue par an.

\section{Gestion des risques}

Il s'agit d'éviter les sinistres par la mise en évidence des sources d'erreurs et des lacunes en matière d'organisation. Le Critical Incident Reporting System (CIRS) est un outil de saisie des incidents qui, grâce à un système électronique accessible à tous, permet d'annoncer des incidents critiques de manière anonyme et individuelle, de les enregistrer et de les soumettre à une procédure d'évaluation. Une étude-pilote lancée en 2001 et 2002 par les cliniques gynécologiques de Suisse a montré des résultats encourageants, incitant de nombreuses autres cliniques à opter pour ce système.

Saisie des données, évaluation des résultats et analyse comparative - statistique de I'AGOS L'Association des cliniques en gynécologie/obstétrique suisses (AGOS) saisit et traite les données concernant le diagnostic, les types de traitement et les résultats de plus de $85 \%$ des cliniques gynécologiques de Suisse. L'anamnèse approfondie de chaque patiente admise dans le domaine ambulatoire ou hospitalier fait l'objet d'une évaluation centralisée. Ainsi, les responsables de clinique peuvent voir où se situent leurs forces et leurs faiblesses et prendre des mesures pour y remédier. Une valeur de référence est attribuée à chaque clinique impliquée dans cette statistique. 